\title{
Maternal and fetal amino acid concentrations and fetal outcomes during pre-eclampsia
}

\author{
R. W. Evans ${ }^{1}$, R. W. Powers ${ }^{2}$, R. B. Ness ${ }^{1}$, L. J. Cropcho ${ }^{3}$, A. R. Daftary ${ }^{2}$, \\ G. F. Harger ${ }^{2}$, R. Vergona ${ }^{1}$ and D. N. Finegold ${ }^{4}$ \\ ${ }^{1}$ Department of Epidemiology, University of Pittsburgh, Pittsburgh, PA 15261, USA; ${ }^{2}$ Magee-Women's \\ Research Institute, Pittsburgh, PA 15213, USA; ${ }^{3}$ Department of Pediatric Endocrinology, Children's \\ Hospital of Pittsburgh, Pittsburgh, PA 15213, USA; and ${ }^{4}$ Department of Pediatrics, University of \\ Pittsburgh, Pittsburgh, PA 15261, USA
}

The pathophysiology of pre-eclampsia is contested, but one hypothesis indicates that it is a heterogeneous condition in which only a subset of affected women bear smallfor-gestational age (SGA) babies. In intrauterine growthrestricted (IUGR) infants, placental transport of amino acids is diminished and the resulting decrease in cordblood amino acid concentrations is thought to contribute to their stunted growth. In contrast, the metabolic syndrome (dyslipidaemia, hyperinsulinaemia, hyperglycaemia, hypertension and obesity) which is associated with high amino acid concentrations is more prevalent in women with pre-eclampsia. The focus of this study was to compare maternal and fetal serum amino acid concentrations during normal pregnancy and pre-eclampsia and to evaluate the associations between the amino acid concentrations and fetal growth. The results indicate that maternal and cord-blood amino acid concentrations were significantly higher in women with pre-eclampsia compared with normal pregnant women and the concentrations were inversely associated with measures of infant growth. Maternal and cord-blood amino acid concentrations were also significantly higher in pre-eclamptic mothers with SGA infants compared with pre-eclamptic mothers whose babies were not SGA. These data indicate that, in contrast to IUGR, pre-eclampsia is associated with enhanced placental amino acid transport or reduced fetal amino acid utilization. Furthermore, the data are consistent with the hypothesis that pre-eclampsia is a heterogeneous disease associated with the metabolic syndrome.

\section{Introduction}

The pathophysiology of pre-eclampsia is not well understood and empirical strategies to prevent this major cause of maternal and infant mortality have been unproductive. A central debate about the genesis of pre-eclampsia is whether the disease invariably involves placental hypoperfusion or has a more heterogeneous pathophysiology (Freidman et al., 1991; Lain and Roberts, 2002). Infants born to pre-eclamptic mothers at term are generally not small for their gestational age (Chesley, 1999). However, when pre-eclamptic mothers deliver pre-term, their babies tend to be smaller than other pre-term babies (Xiong et al., 2002).

Women with early disease, but not necessarily women with pre-eclampsia at term, may have a pre-existing hypermetabolic syndrome, termed the metabolic syndrome (Ness and Roberts, 1996). This metabolic syndrome consists of hypertension in concert with adiposity, dyslipidaemia, hyperglycaemia and hyperinsulinaemia.

Email: rwe2@pitt.edu
Although fetuses of women with the metabolic syndrome appear to be exposed to an excess of circulating nutrients, they may not benefit. Indeed, in pre-eclampsia, nutrient abundance may mark the presence of a predisposition to poor fetal growth.

Amino acid concentrations, which are increased in the metabolic syndrome, serve as one of the main nutrient sources for fetal growth, accounting for 20$40 \%$ of fetal energy requirements (Dicke and Henderson, 1988; Herrera, 2000; Kalhan, 2000). Amino acids are actively transported by the placenta from the maternal to the fetal circulation (Young and Prenton, 1969; Ronzoni et al., 1999; Battaglia and Regnault, 2001; Jansson, 2001). Indeed, the placental and fetal uptake of amino acids is in excess of fetal protein synthesis needs (Battaglia and Regnault, 2001; Jansson, 2001).

This study compared maternal and cord-blood amino acid concentrations during normal pregnancy and preeclampsia and evaluated the associations between amino acid concentrations and fetal growth. Because of the evidence that pre-eclampsia is associated with the metabolic syndrome, it was hypothesized that maternal and 
fetal amino acid concentrations would be increased during pre-eclampsia and, paradoxically, would be highest among pre-eclamptic women bearing smallfor-gestational age (SGA) infants. This would contrast with intrauterine growth-restricted (IUGR) pregnancies in which deficient placental amino acid transport is related to reduced fetal growth (Cetin et al., 1992; Jansson et al., 1998; Paolini et al., 2001).

\section{Materials and Methods}

\section{Subjects}

Fifty-nine nulliparous subjects were recruited (19941997) and gave their informed consent at admission to labour and delivery at Magee-Women's Hospital as part of an ongoing investigation of pre-eclampsia approved by the hospital's institutional review board. Twenty-nine of the women (cases) had pre-eclampsia using the criteria of hypertension, proteinuria, hyperuricaemia and the reversal of hypertension and proteinuria after pregnancy. Hypertension was defined as an absolute blood pressure $>$ 140/90 mmHg after 20 weeks of gestation. Proteinuria was defined as $>300 \mathrm{mg}$ per $24 \mathrm{~h}$ urine collection, $>2+$ on a voided, $>1+$ on a catheterized random urine sample, or a protein:creatinine ratio $>0.3$. Hyperuricaemia was defined as $>1$ SD above usual values at the gestational age at which the sample was obtained (at term $>5.5 \mathrm{mg} \mathrm{dl}^{-1}$ ). Severe pre-eclampsia was defined as absolute blood pressure values $>160 \mathrm{mmHg}$ systolic blood pressure or $>110 \mathrm{mmHg}$ diastolic blood pressure, proteinuria $>4+$ on a voided urine sample, hyperuricaemia or HELLP syndrome. HELLP syndrome included the definition of pre-eclampsia as well as thrombocytopaenia $\left(<100000\right.$ platelets $\left.\mathrm{ml}^{-1}\right)$, serum lactate dehydrogenase $\geqslant 164 \mathrm{U} \mathrm{I}^{-1}$ and serum aspartate aminotransferase $=48 \mathrm{U} \mathrm{I}^{-1}$. Thirty women (controls) had uncomplicated pregnancies. These women had no known medical problems, were normotensive and were without proteinuria or hyperuricaemia throughout the pregnancy. The mothers in the two groups were frequency-matched for age, pre-pregnancy body mass index and race. Fetal gestational age was confirmed using sonography at approximately the twentieth week of gestation. Gestational age at the time of sampling of the maternal blood was noted. For the neonates, values for gestational age, birth weight, birth length, head circumference, sex and whether they were SGA or large-for-gestational age (LGA) were recorded. SGA infants were defined as those who were less than the tenth centile for birth weight corrected for gestational age, sex and race. In a similar way, LGA infants were defined as those who were greater than the ninetieth centile for birth weight corrected for gestational age, sex and race. The incidence of self-reported smoking status for both groups was similar (8 of 29 preeclamptic women and 8 of 30 controls), and none of the subjects in this study reported taking aspirin or antioxidants.

\section{Blood samples}

Maternal and cord-blood serum samples were allowed to stand at room temperature (about $18^{\circ} \mathrm{C}$ ) for $1 \mathrm{~h}$, centrifuged at $2000 \mathrm{~g}$ for $20 \mathrm{~min}$, aliquoted under sterile conditions and then stored at $-80^{\circ} \mathrm{C}$ until they were assayed. The fasting status of the patients who provided samples was not available, and was assumed to be nonfasting.

\section{Amino acid analysis}

Samples were centrifuged through an Amicon centrifuge filter at $800 \mathrm{~g}$ and then combined with norleucine and lithium diluent, $\mathrm{pH} 2.2$ (Pickering Labs, Mountain View, CA). The amino acids were separated by cation exchange chromatography (Pickering Labs) using a foursolvent delivery system (Perkin-Elmer, Wellesley, MA). The amino acids were then derivatized with ninhydrin in a post-column reaction (Pickering amino acid analysis module, AT3000) and detected at $500 \mathrm{~nm}$ (UV-visible detector). The Pickering amino acid standard was used to calibrate the system. Total run time was $180 \mathrm{~min}$. Data are not reported for asparagine, glutamate, serine and threonine in the cord-blood samples as chromatographic interferences prevented the measurement of these amino acids in some samples.

\section{Statistical analysis}

Means and standard errors are reported. A $t$ test was used to compare means. ANOVA, followed by Tukey's test, was used to compare three means. Fisher's Exact test was used to compare the percentages of SGA and LGA among cases and controls. Partial correlations between amino acid concentrations and fetal outcomes were made after adjusting for gestational age. Significance was accepted at $P<0.05$.

\section{Results}

The characteristics of the subjects are shown (Table 1). The mothers in the two groups were frequency-matched for age, body mass index and race; these values were similar. Hypertension is a requirement for the diagnosis of pre-eclampsia, hence, the cases had markedly higher systolic and diastolic blood pressures than controls. The infants in the pre-eclamptic group were on average smaller (weight, length, head circumference) at birth than the infants of control mothers and this is primarily, although not entirely, explained by their earlier gestational age. 
Table 1. Clinical characteristics of the mothers and infants involved in the study

\begin{tabular}{|c|c|c|c|}
\hline & Parameter & Normal pregnancy $(n=30)$ & Pre-eclampsia $(n)$ \\
\hline \multirow[t]{13}{*}{ Mothers } & Age (years) & $24.8 \pm 0.9$ & $27.0 \pm 1.2(27)$ \\
\hline & Body mass index $\left(\mathrm{kg} \mathrm{m}^{-2}\right)$ & $26.2 \pm 1.4$ & $26.2 \pm 1.0(27)$ \\
\hline & Systolic blood pressure $(\mathrm{mmHg})$ & $118.9 \pm 1.8$ & $155.6 \pm 2.8(29)^{*}$ \\
\hline & Diastolic blood pressure $(\mathrm{mmHg})$ & $72.0 \pm 1.1$ & $92.3 \pm 1.5(29)^{*}$ \\
\hline & Serum haemoglobin $\left(\mathrm{g} \mathrm{dl}^{-1}\right)$ & $12.9+0.2$ & $13.3+0.1(24)$ \\
\hline & Hematocrit $(\%)$ & $37.4+0.5$ & $38.2+0.4(24)$ \\
\hline & Urine protein (g per $24 \mathrm{~h}$ urine collection) & $0.24+0.05$ & $1.82+0.21(28)^{*}$ \\
\hline & Severe pre-eclampsia $(n)$ & na & 15 \\
\hline & Race (\%) & & \\
\hline & Caucasian & 76.7 & 75.9 \\
\hline & Black & 23.3 & 20.7 \\
\hline & Other & 0.0 & 3.4 \\
\hline & Sampling age (weeks) & $38.8 \pm 0.6$ & $35.5 \pm 0.5(29)^{*}$ \\
\hline \multirow[t]{9}{*}{ Neonates } & Gestational age (weeks) & $39.5 \pm 0.2$ & $35.5 \pm 0.5(29)^{*}$ \\
\hline & Birth weight (g) & $3576 \pm 75$ & $2456 \pm 152(26)^{*}$ \\
\hline & Birth length $(\mathrm{cm})$ & $51.9 \pm 0.3$ & $46.9 \pm 0.7(24)^{*}$ \\
\hline & Head circumference $(\mathrm{cm})$ & $34.6 \pm 0.2$ & $31.8 \pm 0.5(24)^{*}$ \\
\hline & $\operatorname{SGA}(n)$ & 1 & $8^{\dagger}$ \\
\hline & $\operatorname{LGA}(n)$ & 6 & 2 \\
\hline & $\operatorname{Sex}(n)^{\ddagger}$ & & \\
\hline & Male & 21 & 19 \\
\hline & Female & 9 & 9 \\
\hline
\end{tabular}

Small-for-gestational age (SGA) and large-for-gestational age (LGA) values were compared using Fisher's Exact test. All other comparisons used the $t$ test; ${ }^{*} P<0.001 ;{ }^{\dagger} P<0.01$ compared with normal pregnancy values. ${ }^{\ddagger}$ Sex of one child was not recorded.

\section{Maternal amino acids during pre-eclampsia}

The mean concentration of most maternal amino acids was higher in women with pre-eclampsia compared with controls (Table 2). Results for all individual amino acids required for protein synthesis, except for tryptophan, and their summed total are shown. Ten of the 19 individual amino acids and the total amino acid concentration were significantly higher among pre-eclamptic women. Two of four amino acids that are products of intermediary metabolism (citrulline and 3-methylhistidine) were also higher during pre-eclampsia.

Proteinuria is a clinical feature of pre-eclampsia and could lead to a decrease in maternal amino acid concentrations. However, the results indicated an increase in plasma amino acid concentrations during pre-eclampsia. Furthermore, no significant correlations were observed between the maternal amino acid concentrations and urinary protein concentrations.

The mean total amino acid concentration for the women with pre-eclampsia who had SGA infants $\left(392.8 \pm 49.9 \mu \mathrm{mol} 100 \mathrm{ml}^{-1}\right)$ was significantly increased compared with the pre-eclamptic women who did not have SGA infants $\left(286.6 \pm 9.5 \mu \mathrm{mol} 100 \mathrm{ml}^{-1}\right.$, $P<0.005)$ and compared with controls with non-SGA infants $\left(256.3 \pm 7.0 \mu \mathrm{mol} 100 \mathrm{ml}^{-1}, P<0.001\right)$. The maternal total amino acid concentration in women with pre-eclampsia and non-SGA infants was also significantly increased compared with controls with non-SGA infants $(P<0.02)$.

\section{Cord-blood amino acid concentrations during pre-eclampsia}

Among the 15 normal pregnancy and 16 preeclamptic subjects who had matched cord-blood samples available for this analysis, several amino acids were significantly higher among the infants of preeclamptic mothers compared with the infants of controls (Table 3). These included alanine, lysine, methionine, proline, taurine and total amino acids.

Infants were again divided into either SGA or nonSGA groups and the mean cord-blood total amino acid concentrations were compared. Similar to maternal total amino acid concentrations, mean cord-blood total amino acid concentration in SGA infants from pre-eclamptic women $\left(320.8 \pm 32.5 \mu \mathrm{mol} 100 \mathrm{ml}^{-1}\right)$ was significantly higher compared with the pre-eclamptic women with non-SGA infants $\left(251.9 \pm 16.7 \mu \mathrm{mol} 100 \mathrm{ml}^{-1}\right.$, $P=0.05)$, as well as with the controls with nonSGA infants $\left(231.1 \pm 11.8 \mu \mathrm{mol} 100 \mathrm{ml}^{-1}, P<0.005\right)$. However, cord-blood total amino acid concentrations in non-SGA infants of women with pre-eclampsia were not 
Table 2. Serum amino acid concentrations in normal pregnant women and women with pre-eclampsia

\begin{tabular}{|c|c|c|}
\hline Amino acid & $\begin{array}{l}\text { Normal pregnancy } \\
\qquad(n=30)\end{array}$ & $\begin{array}{l}\text { Pre-eclampsia } \\
\quad(n=29)\end{array}$ \\
\hline \multicolumn{3}{|l|}{ Essential } \\
\hline Arginine & $7.9 \pm 0.4$ & $7.9 \pm 0.5$ \\
\hline Histidine & $8.8 \pm 0.3$ & $11.5 \pm 0.8^{\ddagger}$ \\
\hline Isoleucine & $5.2 \pm 0.3$ & $6.4 \pm 0.3^{\dagger}$ \\
\hline Leucine & $10.4 \pm 0.5$ & $11.9 \pm 0.7$ \\
\hline Lysine & $13.9 \pm 0.7$ & $16.1 \pm 0.9$ \\
\hline Methionine & $2.3 \pm 0.1$ & $3.0 \pm 0.2^{\ddagger}$ \\
\hline Phenylalanine & $6.9 \pm 0.4$ & $8.2 \pm 0.6$ \\
\hline Valine & $15.9 \pm 0.7$ & $19.6 \pm 0.9^{\ddagger}$ \\
\hline \multicolumn{3}{|l|}{ Non-essential } \\
\hline Alanine & $37.2 \pm 1.3$ & $51.1 \pm 5.0^{*}$ \\
\hline Asparagine & $4.9 \pm 0.2$ & $5.5 \pm 0.4$ \\
\hline Aspartic acid & $5.1 \pm 0.4$ & $5.1 \pm 0.4$ \\
\hline Cystine & $2.8 \pm 0.1$ & $3.7 \pm 0.3^{\ddagger}$ \\
\hline Glutamate & $11.4 \pm 1.3$ & $14.9 \pm 2.0$ \\
\hline Glutamine & $42.8 \pm 1.1$ & $52.4 \pm 2.8^{\ddagger}$ \\
\hline Glycine & $20.0 \pm 1.0$ & $23.2 \pm 2.0$ \\
\hline Proline & $12.6 \pm 0.6$ & $16.0 \pm 1.0^{\ddagger}$ \\
\hline Serine & $11.5 \pm 0.5$ & $12.4 \pm 0.8$ \\
\hline Threonine & $16.1 \pm 0.7$ & $19.8 \pm 1.5^{*}$ \\
\hline Tyrosine & $4.5 \pm 0.3$ & $5.9 \pm 0.3^{\ddagger}$ \\
\hline Total & $260.2 \pm 7.8$ & $315.9 \pm 17.3^{\ddagger}$ \\
\hline Citrulline & $1.6 \pm 0.1$ & $2.2 \pm 0.1^{\ddagger}$ \\
\hline 3-Methylhistidine & $0.20 \pm 0.06$ & $0.42 \pm 0.07^{*}$ \\
\hline Ornithine & $4.5 \pm 0.5$ & $5.9 \pm 0.7$ \\
\hline Taurine & $12.2 \pm 0.5$ & $11.3 \pm 0.8$ \\
\hline
\end{tabular}

Values are means $\left(\mu \mathrm{mol} 100 \mathrm{ml}^{-1}\right) \pm \mathrm{SEM} ;{ }^{*} P<0.05 ;{ }^{\dagger} P<0.01$; $\ddagger P<0.005$ ( $t$ test), compared with normal pregnancy values.

significantly different from non-SGA infants of normal pregnant women.

\section{Maternal amino acid concentrations and fetal growth}

Overall, there were consistent inverse relationships between maternal amino acid concentrations and birth weight and birth length of infants after adjustment for gestational age, within both the control subjects and the women with pre-eclampsia. Specifically, during normal pregnancy, the amino acids significantly $(P<0.05)$ negatively correlated with birth weight were the three branched-chain amino acids isoleucine $(r=-0.470)$, leucine $(r=-0.498, P<0.01)$ and valine $(r=-0.458)$, as well as lysine $(r=-0.456)$ and total amino acids $(r=-0.389)$. Valine $(r=-0.470)$, methionine $(r=-0.415)$ and tyrosine $(r=-0.457)$ were significantly and inversely correlated with birth weight during pre-eclampsia. For birth length, during normal pregnancy, isoleucine $(r=-0.388)$ and valine $(r=-0.394)$ were significantly and inversely associated with this outcome. Valine $(r=-0.443)$, asparagine
Table 3. Cord-blood amino acid concentrations in infants of normal pregnant women and women with pre-eclampsia

\begin{tabular}{lcc}
\hline Amino acid & $\begin{array}{c}\text { Normal pregnancy } \\
(n=15)\end{array}$ & $\begin{array}{c}\text { Pre-eclampsia } \\
(n=16)\end{array}$ \\
\hline Essential & & \\
Arginine & $5.1 \pm 0.5$ & $4.0 \pm 0.6$ \\
Histidine & $8.7 \pm 0.9$ & $11.1 \pm 0.9$ \\
Isoleucine & $6.4 \pm 0.5$ & $6.6 \pm 0.3$ \\
Leucine & $11.2 \pm 0.9$ & $10.9 \pm 0.5$ \\
Lysine & $27.2 \pm 2.2$ & $32.8 \pm 1.4^{*}$ \\
Methionine & $2.9 \pm 0.2$ & $3.8 \pm 0.3^{*}$ \\
Phenylalanine & $7.5 \pm 0.4$ & $8.1 \pm 0.6$ \\
Valine & $20.0 \pm 1.1$ & $20.4 \pm 0.6$ \\
Non-essential & & \\
Alanine & $48.9 \pm 3.9$ & $72.3 \pm 9.9^{*}$ \\
Aspartic acid & $1.8 \pm 0.3$ & $4.2 \pm 1.1$ \\
Cystine & $0.4 \pm 0.2$ & $2.1 \pm 0.9$ \\
Glutamine & $45.4 \pm 2.1$ & $48.8 \pm 2.0$ \\
Glycine & $25.1 \pm 1.2$ & $31.5 \pm 4.6$ \\
Proline & $13.5 \pm 0.8$ & $17.1 \pm 1.5^{*}$ \\
Tyrosine & $6.5 \pm 0.5$ & $7.6 \pm 0.5$ \\
Total & $230.6 \pm 11.3$ & $281.4 \pm 17.6^{*}$ \\
Citrulline & $1.01 \pm 0.06$ & $1.19 \pm 0.1$ \\
3-Methylhistidine & $0.22 \pm 0.2$ & $0.53 \pm 0.07$ \\
Ornithine & $11.0 \pm 1.5$ & $10.8 \pm 1.2$ \\
Taurine & $16.5 \pm 1.9$ & $28.1 \pm 3.5^{\dagger}$ \\
\hline Values & & \\
\hline
\end{tabular}

Values are means $\left(\mu \mathrm{mol} 100 \mathrm{ml}^{-1}\right) \pm \mathrm{SEM} ;{ }^{*} P<0.05 ;{ }^{\dagger} P<0.01$ compared with normal pregnancy values.

$(r=-0.604, P<0.005)$, glutamine $(r=-0.422)$ and tyrosine $(r=-0.419)$ were inversely correlated with birth length during pre-eclampsia.

There were no significant correlations between any maternal amino acid and head circumference of the infants among the women with a normal pregnancy outcome after adjusting for gestational age (Table 4). However, among the women with pre-eclampsia, several amino acids showed significant inverse correlations with head circumference: asparagine $(r=-0.636$, $P<0.005)$, methionine $(r=-0.564, P<0.01)$, tyrosine $(r=-0.553, P<0.01)$, valine $(r=-0.494, P<0.05)$ and total amino acids $(r=-0.465, P<0.05)$. For three of these amino acids (asparagine, tyrosine and valine), correlations with head circumference were also significantly different when comparing the normal and pre-eclamptic groups. The relationship between head circumference of the infant and total amino acids (Fig. 1) is representative of the significant associations found for individual amino acids. At low amino acid concentrations the head circumferences were similar for both groups, but as maternal amino acid concentrations increased, the head circumference values of infants for the controls remained constant, whereas they decreased for the pre-eclamptic women. 
Table 4. Correlation ( $r$ ) of maternal amino acid concentration with head circumference, adjusted for gestational age, of infants of normal pregnant women and women with pre-eclampsia

\begin{tabular}{lrc}
\hline Amino acid & $\begin{array}{c}\text { Normal pregnancy } \\
(n=30)\end{array}$ & $\begin{array}{c}\text { Pre-eclampsia } \\
(n=24)\end{array}$ \\
\hline Essential & -0.107 & -0.042 \\
Arginine & 0.098 & -0.363 \\
Histidine & -0.202 & -0.167 \\
Isoleucine & -0.129 & -0.366 \\
Leucine & -0.127 & $-0.347^{\S}$ \\
Lysine & -0.060 & $-0.564^{\dagger}$ \\
Methionine & 0.148 & $-0.257^{\S}$ \\
Phenylalanine & -0.116 & $-0.494^{*}$, \\
Valine & & \\
Non-essential & 0.090 & -0.338 \\
Alanine & 0.021 & $-0.636^{\ddagger, \S}$ \\
Asparagine & 0.093 & -0.110 \\
Aspartic acid & -0.352 & -0.078 \\
Cystine & 0.007 & -0.022 \\
Glutamate & -0.104 & -0.377 \\
Glutamine & 0.151 & -0.348 \\
Glycine & 0.174 & $-0.238^{\S}$ \\
Proline & 0.077 & -0.209 \\
Serine & -0.134 & $-0.489^{*}$ \\
Threonine & 0.079 & $-0.553^{\dagger,}$ \\
Tyrosine & -0.006 & $-0.465^{*}$ \\
Total & 0.036 & -0.099 \\
Citrulline & 0.075 & 0.027 \\
Ornithine & -0.019 & 0.033 \\
Taurine & & $c 019$ \\
\hline & & \\
\hline
\end{tabular}

${ }^{*} P<0.05 ; \dagger P<0.01 ;{ }^{\ddagger} P<0.005$ ( $r$ values), partial correlations; $\S P<0.05 ; \rrbracket P<0.005$ (comparison of the relationship between head circumference and amino acid concentrations among pre-eclamptic and normal pregnancies; slopes and intercepts compared simultaneously).

\section{Discussion}

The main findings of this study are: (1) maternal and cord-blood amino acid concentrations were significantly increased during pre-eclampsia; (2) the smaller the infant, the higher the maternal amino acid concentrations, a relationship that, for head circumference, was restricted to pre-eclamptic pregnancies; and (3) pre-eclamptic women with SGA infants had higher maternal and cordblood amino acid concentrations than women without SGA (pre-eclamptics or controls), whereas the cordblood amino acid concentrations in pre-eclamptic and control women without SGA did not differ.

The largest differences in mean maternal amino acid concentrations between the pre-eclamptic women and controls were for alanine and glutamine $(13.9 \mu \mathrm{mol}$ $100 \mathrm{ml}^{-1}$ and $9.6 \mu \mathrm{mol} 100 \mathrm{ml}^{-1}$, respectively), and these are the most important amino acids for gluconeogenesis (Stumvoll et al., 1999). These amino acids could fuel the hyperglycaemia characteristic of the metabolic syndrome.

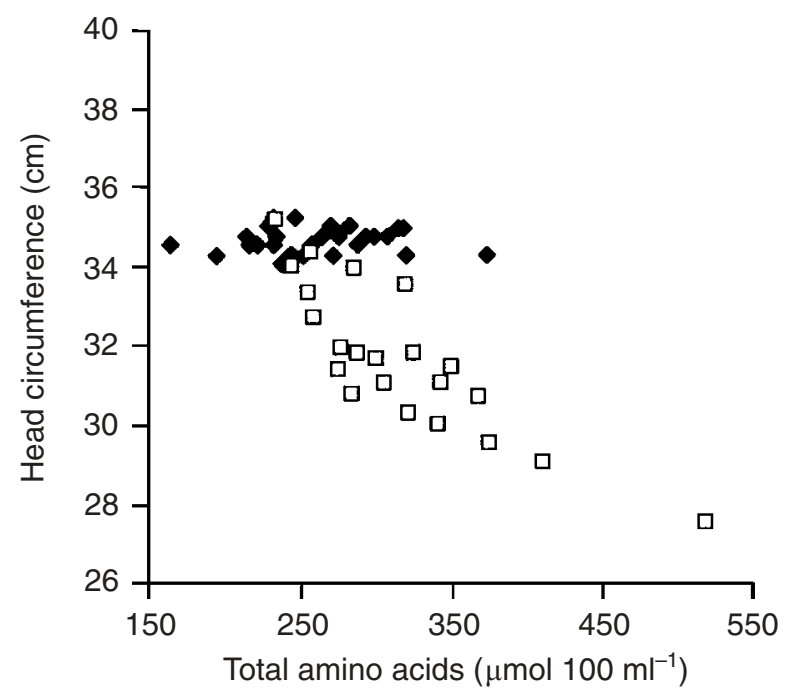

Fig. 1. Head circumference of infants adjusted for gestational age versus total serum amino acid concentration of normal pregnant women ( $\square$ ) and pre-eclamptic women ( $\square$ ).

Two previous studies, consistent with this report, found increased maternal amino acid concentrations during pre-eclampsia (Cockburn et al., 1971; Li et al., 1996). A third study reported significantly lower maternal amino acid concentrations during pre-eclampsia (D'Aniello et al., 2001). However, it is noteworthy that the amino acid values reported in this third study were two orders of magnitude higher than those generally observed.

The present findings provide new insight into the interrelationships among amino acid concentrations, preeclampsia and fetal growth, because they indicate that the most severely growth-restricted babies were exposed, via cord blood, to the highest amino acid concentrations. In particular, reduced head circumference, the most preserved fetal growth parameter, was uniquely related to high amino acid concentrations in pre-eclamptic women. Moreover, pre-eclamptic women with SGA babies had higher amino acid concentrations than controls but pre-eclamptic women whose babies were not SGA did not have higher amino acid concentrations. These observations are consistent with the hypothesis that pre-eclampsia does not involve one uniform pathophysiological process. The subset of women with pre-eclampsia having poor fetal outcomes may have the metabolic syndrome (Lain and Roberts, 2002), a syndrome characterized by increased amino acid concentrations (Pijl et al., 1994).

These findings contrast with observations in IUGR pregnancies wherein maternal amino acid concentrations are generally not different from controls and cordblood amino acid concentrations are lower due to reduced placental amino acid transport (Harrington et al,. 1999; Bajoria et al., 2001; Paolini et al., 2001; Jansson 
et al., 2002). Many transport systems for amino acids, including A, ASC and L, have been identified within the human placenta (Jansson, 2001). There is considerable redundancy between the systems and competition for a carrier within a system. SGA babies from pre-eclamptic pregnancies may have increased placental amino acid transport or they may have aberrant utilization. The cordblood measurements in the present study represented mixed arterial and venous cord blood; thus the cordblood concentrations provide limited information about what the fetus utilized. Further limitations of this study include the retrospective design, the small sample size and the unclear fasting status of the subjects who provided the samples. Multiple comparisons were made but this restriction is mitigated by the number of differences observed that were significant at $P<0.005$. The gestational age at sampling was also different for the two groups of pregnant women. However, amino acid concentrations did not change during the sampling range studied (data not shown). In a similar way, adjusting the amino acid concentrations for sampling age does not alter the conclusions reached.

In conclusion, the results of this study emphasize the differences in maternal and fetal amino acid concentrations observed between pre-eclamptic and normal pregnancies and the divergent associations between amino acid concentrations and fetal outcome in the two groups. In particular, the concentrations of several amino acids were inversely correlated with head circumference of infants in pre-eclamptic pregnancies but not in normal pregnancies. In addition, total amino acid concentrations were significantly higher in maternal and cord blood of pre-eclamptic women with SGA infants compared with either pre-eclamptic or control women without SGA infants. This result is intriguing, as it contrasts with the observation that cord-blood amino acid concentrations are reduced in IUGR infants. These data provide support for the notion that pre-eclampsia is a heterogeneous disorder associated with a hypermetabolic state wherein, paradoxically, the more abundant the availability of amino acids to the placenta, the less the fetus grows.

The authors thank J. Roberts for providing data and samples from his ongoing studies of pre-eclampsia (P01 HD30367).

\section{References}

Bajoria R, Sooranna SR, Ward S, D'Souza S and Hancock M (2001) Placental transport rather than maternal concentration of amino acids regulates fetal growth in monochorionic twins: implications for fetal origin hypothesis American Journal of Obstetrics and Gynecology 185 1239-1246

Battaglia FC and Regnault TR (2001) Placental transport and metabolism of amino acids Placenta 22 145-161

Cetin I, Marconi AM, Corbetta C, Lanfranchi A, Baggiani AM, Battaglia FC and Pardi G (1992) Fetal amino acids in normal pregnancies and in pregnancies complicated by intrauterine growth retardation Early Human Development 29 183-186

Chesley L (1999) Chesley's Hypertensive Disorders in Pregnancy Appleton \& Lange, Stamford
Cockburn F, Blagden A, Michie EA and Forfar JO (1971) The influence of pre-eclampsia and diabetes mellitus on plasma free amino acids in maternal, umbilical vein and infant blood Journal of Obstetrics and Gynecology of the British Commonwealth 78 215-231

D'Aniello G, Tolino A and Fisher G (2001) Plasma L-arginine is markedly reduced in pregnant women affected by pre-eclampsia Journal of Chromatography B Biomedical Sciences and Applications 753 427-431

Dicke JM and Henderson GI (1988) Placental amino acid uptake in normal and complicated pregnancies American Journal of the Medical Sciences $295223-227$

Freidman SA, Taylor RN and Roberts JM (1991) Pathophysiology of pre-eclampsia Clinics in Perinatology 18 661-682

Harrington B, Glazier J, D'Souza S and Sibley C (1999) System A amino acid transporter activity in human placental microvillous membrane vesicles in relation to various anthropometric measurements in appropriate and small for gestational age babies Pediatric Research 45 810-814

Herrera E (2000) Metabolic adaptations in pregnancy and their implications for the availability of substrates to the fetus European Journal of Clinical Nutrition 54 Supplement 1 47-51

Jansson T (2001) Amino acid transporters in the human placenta Pediatric Research 49 141-147

Jansson T, Scholtbach V and Powell TL (1998) Placental transport of leucine and lysine is reduced in intrauterine growth restriction Pediatric Research 44 532-537

Jansson T, Ekstrand Y, Bjorn C, Wennergren M and Powell TL (2002) Alterations in the activity of placental amino acid transporters in pregnancies complicated by diabetes Diabetes 51 2214-2219

Kalhan SC (2000) Protein metabolism in pregnancy American Journal of Clinical Nutrition 71 Supplement 5 1249-1255

Lain KY and Roberts JM (2002) Contemporary concepts of the pathogenesis and management of pre-eclampsia Journal of the American Medical Association 287 3183-3186

Li J, Sun J and Lu H (1996) Determination of serum free amino acids and ammonia in pregnant women with severe pregnancy induced hypertension Chinese Journal of Obstetrics and Gynecology 31468 470

Ness RB and Roberts JM (1996) Heterogeneous causes constituting the single syndrome of pre-eclampsia: a hypothesis and its implications American Journal of Obstetrics and Gynecology 175 1365-1370

Paolini CL, Marconi AM, Ronzoni S, Di NM, Fennessey PV, Pardi G and Battaglia FC (2001) Placental transport of leucine, phenylalanine, glycine, and proline in intrauterine growth-restricted pregnancies Journal of Clinical Endocrinology and Metabolism 86 5427-5432

Pijl H, Potter VLBJ, Toornvliet AC, Radder JK, Onkenhout W, Frolich M and Meinders AE (1994) Insulin-induced decline of plasma amino acid concentrations in obese subjects with and without noninsulin-dependent diabetes Metabolism: Clinical and Experimental 43 640-646

Ronzoni S, Marconi AM, Cetin I, Paolini CL, Teng C, Pardi G and Battaglia FC (1999) Umbilical amino acid uptake at increasing maternal amino acid concentrations: effect of a maternal amino acid infusate American Journal of Obstetrics and Gynecology 181 477-483

Stumvoll M, Perriello G, Meyer C and Gerich J (1999) Role of glutamine in human carbohydrate metabolism in kidney and other tissues Kidney International 55 778-792

Xiong X, Demianczuk NN, Saunders LD, Wang FL and Fraser WD (2002) Impact of pre-eclampsia and gestational hypertension on birth weight by gestational age American Journal of Epidemiology 155 203-209

Young M and Prenton MA (1969) Maternal and fetal plasma amino acid concentrations during gestation and in retarded fetal growth Journal of Obstetrics and Gynecology of the British Comonwealth 76 333-344

Received 10 December 2002.

First decision 6 February 2003.

Revised manuscript received 4 March 2003.

Accepted 5 March 2003. 